\title{
The Paranasal Sinuses as a Protective Crumple Zone for the Orbit
}

Robert M. Kellman, MD; Christopher Schmidt, MD

Objectives/Hypothesis: The purpose of this study is to test the theory that the paranasal sinuses serve a protective function for the central nervous system and special sensory organs.

Study Design: Nonrandomized experimental trauma study with fresh human cadavers.

Methods: Fresh human cadaver heads were obtained and the sinuses on one side underwent endoscopic endonasal sinus surgery and were then filled with radio-opaque bone cement to obliterate them. The contralateral sinuses were not operated upon to allow both for comparison to the experimental side and to serve as an intraspecimen control. The cadavers underwent serial computed tomography (CT) scans. Scans were performed prior to surgery, after surgery, and after unilateral sinus obliteration to obtain baseline CT studies prior to any impact testing. Sequential drops of increasing energy were then performed directing the impacts onto the globes. Initial endpoints were either orbital fractures or ocular injury. Trauma was induced by impacting a weighted rod onto the globes using a guided drop technique. Orbital rim impact was avoided, so that the effect of direct globe trauma could be assessed; fractures were thus induced via the hydraulic mechanism, in which force is transmitted through the globe to the surrounding tissues and orbital walls. After initial injury endpoints were met, additional impact testing was performed on globes, in which fractures occurred with lower drop forces to ensure impact energy equivalence between the control and the experimental sides.

Results: All the experimentally obliterated paranasal sinus orbits tested suffered trauma-induced globe ruptures, and no orbital wall fractures were encountered. On the control sides, no globe ruptures occurred at either an equivalent or higher energy

From the Department of Otolaryngology and Communication Sciences, State University of New York Upstate Medical University, Syracuse, New York, U.S.A 14,2009

Editor's Note: This Manuscript was accepted for publication May

Send correspondence to Robert M. Kellman, MD, Professor and Chair, Department of Otolaryngology and Communication Sciences, SUNY Upstate Medical University, 750 E. Adams Street, Syracuse, New York 13210. E-mail: kellmanr@upstate.edu

DOI: 10.1002/lary.20583 than the energy needed to induce globe ruptures on the experimental side orbits, although orbital floor fractures on the control sides occurred after lower energy impacts in some cases.

Conclusions: This study demonstrates that the thin orbital floor fractures preferentially, thereby protecting the globe from rupture as a result of the directed trauma. When the sinus crumple zones were eliminated, globe ruptures occurred.

Key Words: Paranasal sinuses, crumple zones, globe rupture, protective effect, facial trauma.

Laryngoscope, 119:1682-1690, 2009

\section{INTRODUCTION}

People have long debated the function of the paranasal sinuses. Various authors have described structural and/or functional theories for the existence of the sinuses, some more plausible than others. The list of proposed paranasal sinus functions derived from varied publications ${ }^{1-5}$ has included: 1) the reduction of cranial weight, 2) accommodation for the developmental increase in the ratio of body:head size, 3) the replacement of functionless bone, 4) interspaces between developing cranial architecture, 5) ventilation, 6) the secretion of mucus to moisten the olfactory mucosa, 7) respiratory (heating and humidification) functions, 8) shock absorption spaces, 9) insulation from heated or cold environments, 10) a resonance chamber for voice, 11) vestigial (or active) olfactory organ space from evolutionary development, 12) flotation device, and 13) a source of immunoglobulins to bolster immune defenses.

These theories have remained exactly that over time, untested hypotheses. Some theories have been scientifically discredited, such as mucus secretion, use as an air reservoir, olfactory enhancement, and resonance chamber function, but no clear remaining theory predominates. Authors theorizing about the remaining possible functions have conceded the wholesale lack of empiric evidence, all the while attempting to use fossil records, species development, and evolution to give more credence to particular developmental theories.

Proetz in 1953 (as quoted by Blaney ${ }^{3}$ ) commented that the paranasal sinuses have "aroused more speculation than investigation." Blaney, ${ }^{3}$ in 1990, referred to 
the deficiency of plausible arguments and the "lack of rigorous scientific evidence" for the existence of the paranasal sinuses. More recently, Rae and Koppe, ${ }^{1}$ in 2004 , analyzed fossil records in the development of catarrhines in an attempt to detail the evolutionary anthropology behind the development of the paranasal sinuses. In reviewing experimental data regarding bite forces and strain data of the paranasal structures in primate ancestry, they commented that "experiments clearly indicate that bony structures of the facial skeleton cannot be understood solely as a means of countering masticatory stress, leaving the association between paranasal sinus morphology and biomechanical forces open to question."1

In the most recent review, Keir $(2009)^{5}$ performed an extensive literature review and discussed the validity of each theory. Each theory was theoretically or scientifically refuted, except the hypothesis that the sinuses function to enhance immune and antimicrobial function.

The hypothesis of this study is that the paranasal sinuses provide a survival benefit serving to protect surrounding vital organs, such as the eyes, optic nerves, and brain by acting as "crumple zones" 6 for trauma to the area. Crumple zones function to decrease impact energy to areas around them. Crumple zones, sometimes also known as "crash zones," usually provide both a distance buffer from an initial impact allowing for deceleration, and they allow for the dispersion of kinetic energy. More importantly, crumple zones provide a compressible or fragmentable barrier that absorbs and disperses energy through the destruction and/or deformation of the crumple zone itself. The time for energy transfer and the distance involved with impact are both increased as the impact energy passes through a crumple zone. This in turn decreases the net energy and force transferred to a protected area in an impact. Thus, the crumple zone serves as a passive protective barrier.

Common everyday examples of the function of crumple zones are seen in automobile accidents. Passengers in the main cabin of many automobiles are surrounded by crumple zones. In a simplified example, a front-end collision results in the telescoping of a relatively collapsible front of the automobile without intrusion into the passenger compartment. Automobile crumple zones yield to the impact, thereby dispersing and decreasing the energy and forces transmitted to the passenger area and the occupants of the vehicle. Force and impulse transmitted to the passenger compartment are decreased, leading to less injury to the occupants at the expense of crumple zone destruction, i.e., the passengers survive, but the car is "totaled."

In the face and head, it is here theorized that the paranasal sinuses act as crumple zones to protect organs that are needed for survival. It is well known that the facial architecture includes thick buttress areas surrounded by thin, nonsupportive bone. ${ }^{7,8}$ When repairing facial fractures, in fact, it is important to re-establish these buttress areas, whereas the structural integrity of the surrounding thin areas is considered less important. If the bone between the buttresses were more solid, fractures would probably occur less frequently, and they would be technically easier to repair. However, the facial bones would also be less likely to crumple, and thus more force and energy would be directly transmitted to the eyes and brain. The concept of the maxillofacial bony structure potentially acting as a shock absorber to protect the brain in trauma was suggested by Rene LeFort in 1901 (as translated by Tessier in 1972). ${ }^{9} \mathrm{He}$ suggested that "a great number of weak points...cause the facial bones to break up into fragments so that stress is exhausted...preserving the integrity of the bony envelope of the brain."

It has been previously suggested ${ }^{10}$ that the location of each of the paranasal sinuses allows for protection of different areas and vital structures. The maxillary sinuses would primarily protect the globes, middle cranial fossae, and the optic nerves. The ethmoid sinuses would protect the globes, optic, olfactory nerves, and both the anterior and middle cranial fossae. The sphenoid sinuses would be protective of the optic nerves, carotid arteries, the cavernous sinuses, and pituitary gland, and the frontal sinuses would minimize frontal lobe trauma from direct frontal impact.

Although this theory is intriguing, testing these possible protective functions of the sinuses is technically challenging, both due to the difficulty of eliminating the sinuses without altering the underlying anatomy, and the challenge of measuring the forces transmitted and the injury created to the surrounding vital structures as the result of an experimental impact. Additionally, there needed to be available methods to access the sinuses and obliterate them without otherwise destroying the native anatomy. The advent of endoscopic sinus techniques combined with the availability of injectable bone cements with properties that mimic bone provided the technological advances that made this study feasible.

After considering the various sinuses and structures, it was decided that the globe would provide the most accessible structure for impact analysis, and the blowout fracture of the orbit therefore seemed like a reasonable paradigm for the initial testing of the crumple zone theory. It is well known that the frequency of globe injury is low compared with that of orbital trauma severe enough to cause fracture, and serious ocular injury and blindness after such trauma is rare. ${ }^{11-18}$

\section{MATERIALS AND METHODS}

Nine fresh unfixed cadaveric human heads were used in this study. Institutional review board approval for the study was sought and exemption was received. Cadaver heads first underwent computed tomography (CT) scanning in the three main orthogonal planes (axial, coronal, and sagittal) to obtain baseline anatomy using a facial fracture trauma protocol. Axial scans were obtained of $2.5-\mathrm{mm}$ and $0.63-\mathrm{mm}$ thickness. Coronal and sagittal reconstructions from the thinnest slice axial scans were performed, as were 3-dimensional digital reconstructions. The average age of the cadavers was $76.1 \pm 12.2$ years.

Each cadaver underwent unilateral endoscopic transnasal sinus surgery leaving the contralateral sinuses untouched. Surgery consisted of sphenoidotomy, mega antrostomy of the maxillary sinus, and a complete ethmoidectomy including opening of the frontal recess if needed. Repeat CT scanning was then performed to make certain that no iatrogenic injury to the skull base or orbit was created by the surgery. 




Fig. 1. Plastic head specimen secured with 3-point fixation.

Obliteration of the operative sinus cavities was then performed by filling them endoscopically and endonasally with a radio-opaque bone cement. The cement was a two-part self-curing cement composed of polymethylmethacrylate and methylmethacrylate with benzoyl peroxide and polymethylnitrotoulene as activators and initiators of the reaction. Zirconium oxide was added to render the cement opaque.

The bone cement was mixed, injected into, and packed into the experimental sinus cavities created. Packing of the sinuses with the cement before it was completely set was done endonasally with sinus instruments under endoscopic visualization. Triplanar CT scans were then repeated to determine the degree of sinus obliteration by the cement and to ensure that the maxillary, ethmoid, and sphenoid sinuses were filled with cement.

Attempts to pack cement into the frontal sinus from the facial recess were made realizing that this would have limited success. All sinuses, excepting the frontal sinuses, were essentially filled. (Trephination of the frontal sinuses with subsequent filling and obliteration was considered, but in order to minimize possible iatrogenic trauma to the orbit, this was not done.)

The unoperated sides served as intraspecimen controls. Decisions regarding sidedness of paranasal obliteration were nonrandom and were made based on evaluation of the baseline $\mathrm{CT}$ scans for the degree of ease for endoscopic surgery, i.e., the side with the least septal deflection, turbinate hypertrophy, and less-obstructed anatomy was selected for surgical obliteration. After repeat CT scanning, the success of sinus obliteration was analyzed and noted.

One specimen with orbital penetration by the bone cement was excluded from the study. This sample and a fixed head were utilized as test models for the experimental design and weight drop set-up. A total of eight thawed fresh specimens were judged adequate for experimental use. Four specimens had the right sinuses obliterated and four had the left sinuses obliterated.

The cadaveric globes were reinflated to normotonic intraocular pressures (IOPs) (15 to $20 \mathrm{~mm} \mathrm{Hg}$ ) with an intravitreal isotonic saline solution injection to alleviate hypotony. Pressure was checked with either: 1) an invasive (vascular) pressure monitoring system utilizing a 30-gauge needle inserted temporally into the globe and attached to a pressure transducer, or 2) a TONO-PEN XL (Reichert Inc., Depew, NY) digital handheld tonometer, or 3) both. (When both methods were utilized to determine IOPs, agreement was noted.) The eyelids were loosely closed with a temporary tarsorrhaphy suture. (Prelimi- nary testing of our experimental model with the eyelids open resulted in a $100 \%$ globe rupture rate.)

The cadaver heads were then secured with three-point fixation in a halo device attached to a suspension drop tower (Fig. 1). A 3/4-inch diameter, 1.2-meter length polyvinyl chloride (PVC) pipe was then oriented directly over the closed eyelids and globe (Fig. 2). This allowed for a directed perpendicular free fall impact onto the globe without impact upon the orbital rims. A 1.3-mm diameter solid metal rod of either 200- or 300-g mass was dropped from a known height onto the globe. Holes drilled through the PVC pipe every $3 \mathrm{~cm}$ facilitated positioning of the impaction rod and assured height consistency.

The collision of a metallic cylinder onto the globe without impact on the orbital rims can cause an orbital fracture if the energy of impact is sufficient. Kinetic energy (KE) was calculated from the predrop resting gravitational potential energy (GPE), assuming negligible energy loss from friction during the drop. Potential energy was calculated from the mass of the rod dropped and the height of the drop:

$$
\mathrm{KE}=\mathrm{GPE}=\mathrm{mgh}
$$

where $\mathrm{m}=$ mass in grams, $\mathrm{g}=$ gravitational acceleration estimated at $9.80 \mathrm{~m} / \mathrm{s}^{2}$, and $\mathrm{h}=$ height of the drop in meters.

After each drop, heads were evaluated for expected injury patterns, which included ocular injuries (particularly ruptured globe), soft tissue injury and fractures. If no audible injury (cracking of bone) was clearly identified during the drop and palpation of the orbit with a small blunt probe revealed an intact orbit, a repeat impact with a greater energy was performed. Heights were increased in $15-\mathrm{cm}$ steps to generate calculable energy increases. Holes drilled through the PVC piping every $3 \mathrm{~cm}$ facilitated positioning of the impaction rod and assured height consistency. When the maximum height drop was reached using the 200 -g rod at $1.05 \mathrm{~m}(2.06 \mathrm{~J})$ and no endpoint was achieved, the 300 -g rod was then utilized starting at a height of $75 \mathrm{~cm}(2.205 \mathrm{~J})$.

If an orbital fracture was suspected, further impaction was halted until a CT scan of the orbit provided confirmation of its status. Another experimental endpoint besides suspected or actual orbital fracture was frank globe rupture. Injuries short of frank globe ruptures and CT-diagnosed blowout fractures

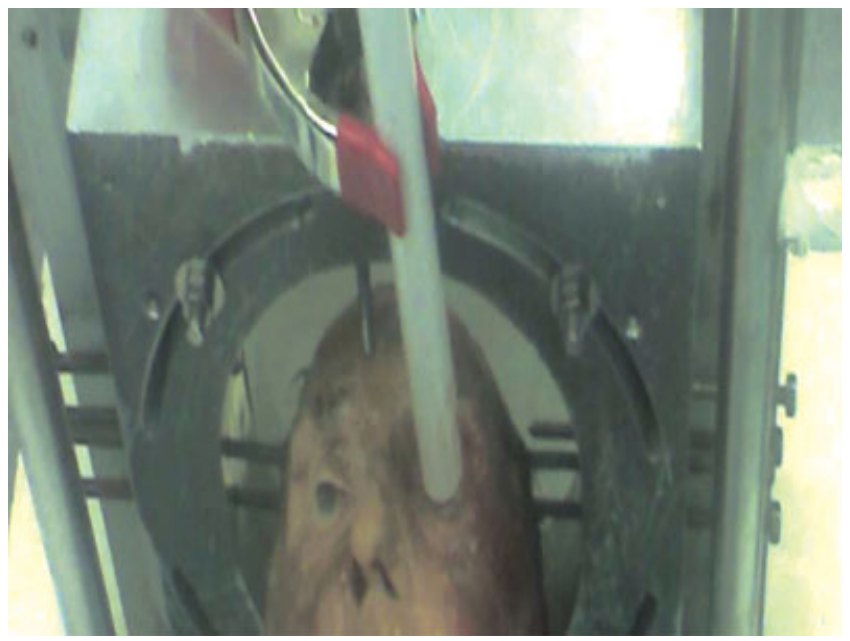

Fig. 2. Cadaver head secured with polyvinyl chloride pipe directed over the globe preventing orbital rim contact during induced trauma. 


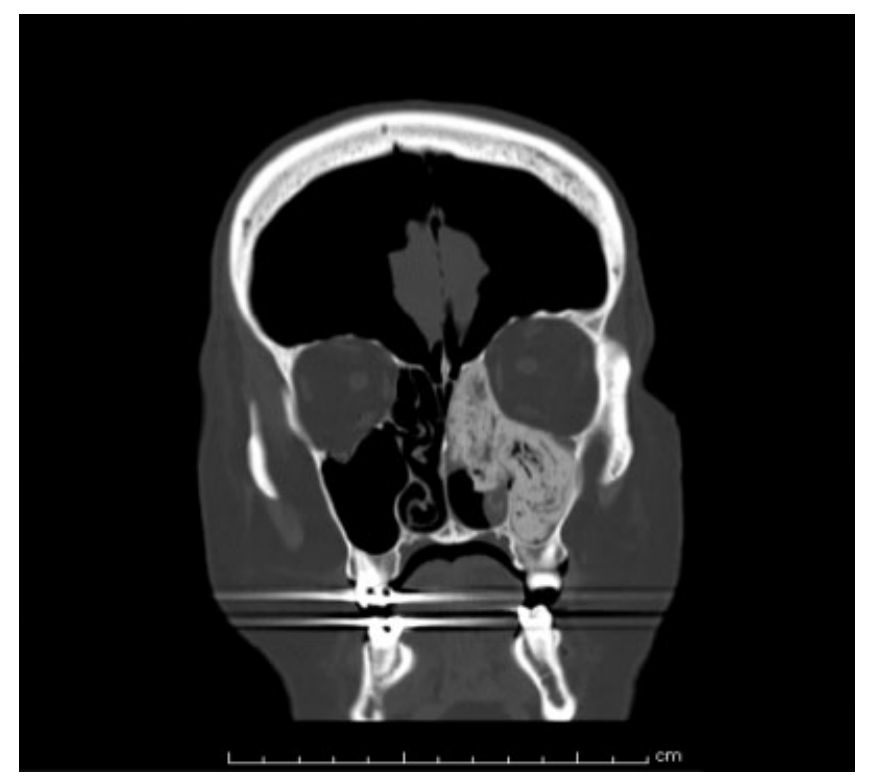

Fig. 3. Comparison the average control globe peak intraocular pressure (IOP) was $116.8 \pm 6.1 \mathrm{~mm} \mathrm{Hg}$. In some cases for technical reasons, peak IOP could not be measured.

mandated further impacts at sequentially higher energies. As needed, further intravitreous saline injection was performed to allow for re-establishment of normotonic IOP prior to further impacts. When available, continuous invasive IOP monitoring was displayed on a monitor and peak impact pressure generated during impact was noted.

In seven of the eight control sides, fractures occurred at lower or equivalent energies to the experimental side end point of globe rupture. Drops after CT confirmation of fractures on the control sides were continued in four heads to generate energy delivery levels exceeding levels that created globe ruptures on the experimental side, to assure that equal or greater energy could be delivered to the globes that did not rupture.

\section{Statistical Analysis}

Because results were all or none with either orbital floor fractures on the control sides or globe ruptures on the experimental sides, no statistical analysis was performed on the data generated. Means and standard deviations of biometric data were calculated using standard spreadsheet software.

\section{RESULTS}

Analysis of the CT scans of the cadavers was performed after sinus surgery looking for any iatrogenic injury to the orbit and skull base, and again after the sinuses were filled to evaluate the degree of filling. The majority of the area of the ethmoid, sphenoid, and maxillary sinuses was filled in all cadavers. Small filling gaps were seen in areas judged to be insignificant relative to the experimental paradigm. The most difficult areas to obliterate by filling were the anterolateral and inferolateral extremes of the maxillary sinuses. Filling of the frontal sinuses, as expected, was very limited.

After repeat impactions to the point of globe rupture on the experimental sides, CT scan analysis revealed no fractures in any of the orbital walls associated with these cement filled sinuses. On the control sides, CT scans confirmed suspected fractures in the orbital floors only (Fig. 3). No medial wall fractures were identified in any orbit, even when impacted to suprathreshold energy levels, with the threshold level determined by the energy level that caused globe rupture on the experimental side.

The impact energies delivered to the cadaver orbits are summarized in Tables I and II. Table I shows the initial experiments with sequentially increased energy delivery until orbital fracture or ocular injury occurred. As shown, all of the sides with experimentally filled sinuses suffered globe ruptures without orbital fractures. The average energy delivered was $1.95 \pm 0.37 \mathrm{~J}$. The control side orbits all suffered orbital floor fractures without globe ruptures. The average energy delivered was $1.65 \pm 0.27 \mathrm{~J}$. Impact peak IOPs were measured in some globes as well. The average peak IOP for the experimental globes (which all ruptured) was $121.5 \pm$ $6.0 \mathrm{~mm} \mathrm{Hg}$. Figure 3 is a representative CT image showing a cadaver head postimpact with the left-side sinuses obliterated, and unoperated right side sinuses with an induced right-sided blowout fracture.

TABLE I.

Peak Energy Delivery at Globe Rupture or Orbital Fracture.

\begin{tabular}{|c|c|c|c|c|c|c|c|c|}
\hline \multirow[b]{2}{*}{ Cadaver } & \multicolumn{4}{|c|}{ Experimental Sinus Side (Bone Cement-Filled Side) } & \multicolumn{4}{|c|}{ Control Sinus Side (Nonfilled Side) } \\
\hline & $\begin{array}{l}\text { Impact Energy } \\
(\mathrm{J})\end{array}$ & $\begin{array}{l}\text { Globe } \\
\text { Rupture }\end{array}$ & $\begin{array}{l}\text { Orbital } \\
\text { Fracture }\end{array}$ & IOP $(\mathrm{mm} \mathrm{Hg})$ & $\begin{array}{l}\text { Impact Energy } \\
\text { (J) }\end{array}$ & $\begin{array}{l}\text { Globe } \\
\text { Rupture }\end{array}$ & $\begin{array}{l}\text { Orbital } \\
\text { Fracture }\end{array}$ & $\begin{array}{c}\mathrm{IOP} \\
(\mathrm{mm} \mathrm{Hg})\end{array}$ \\
\hline 1 & 2.205 & Y & $\mathrm{N}$ & N/A & 1.47 & $\mathrm{~N}$ & Y & $\mathrm{N} / \mathrm{A}$ \\
\hline 2 & 1.764 & Y & $\mathrm{N}$ & $\mathrm{N} / \mathrm{A}$ & 1.47 & $\mathrm{~N}$ & Y & $\mathrm{N} / \mathrm{A}$ \\
\hline 3 & 1.764 & Y & $\mathrm{N}$ & $\mathrm{N} / \mathrm{A}$ & 1.764 & $\mathrm{~N}$ & Y & 108 \\
\hline 4 & 1.764 & Y & $\mathrm{N}$ & $\mathrm{N} / \mathrm{A}$ & 2.058 & $\mathrm{~N}$ & Y & 118 \\
\hline 5 & 1.764 & Y & N & 118 & 1.47 & $\mathrm{~N}$ & Y & 125 \\
\hline 6 & 1.47 & Y & $\mathrm{N}$ & 115 & 1.47 & $\mathrm{~N}$ & Y & $\mathrm{N} / \mathrm{A}$ \\
\hline 7 & 2.646 & Y & $\mathrm{N}$ & 128 & 1.47 & $\mathrm{~N}$ & Y & 115 \\
\hline 8 & 2.205 & Y & $\mathrm{N}$ & 125 & 2.058 & $\mathrm{~N}$ & Y & 118 \\
\hline
\end{tabular}

$\mathrm{IOP}=$ intraocular pressure; N/A = not applicable. 
TABLE II.

Reimpaction Energies to Control Side Globes Compared With Experimental Side.

\begin{tabular}{|c|c|c|c|c|c|c|c|c|}
\hline \multirow[b]{2}{*}{ Cadaver } & \multicolumn{4}{|c|}{ Experimental Sinus Side (Bone Cement-Filled Side) } & \multicolumn{4}{|c|}{ Control Sinus Side (Nonfilled Side) } \\
\hline & $\begin{array}{c}\text { Impact Energy } \\
(\mathrm{J})\end{array}$ & $\begin{array}{l}\text { Globe } \\
\text { Rupture }\end{array}$ & $\begin{array}{l}\text { Orbital } \\
\text { Fracture }\end{array}$ & $\begin{array}{c}\text { IOP } \\
(\mathrm{mm} \mathrm{Hg})\end{array}$ & $\begin{array}{l}\text { Impact Energy } \\
(\mathrm{J})\end{array}$ & $\begin{array}{l}\text { Globe } \\
\text { Rupture }\end{array}$ & $\begin{array}{l}\text { Orbital } \\
\text { Fracture }\end{array}$ & $\begin{array}{c}\mathrm{IOP} \\
(\mathrm{mm} \mathrm{Hg})\end{array}$ \\
\hline 3 & 1.764 & Y & $\mathrm{N}$ & N/A & 1.764 & $\mathrm{~N}$ & Y & 108 \\
\hline 4 & 1.764 & Y & $\mathrm{N}$ & N/A & 2.058 & $\mathrm{~N}$ & Y & 118 \\
\hline $5^{\star}$ & 1.764 & Y & $\mathrm{N}$ & 118 & 2.058 & $\mathrm{~N}$ & Y & N/A \\
\hline $6^{*}$ & 1.47 & $\mathrm{Y}$ & $\mathrm{N}$ & 115 & 1.764 & $\mathrm{~N}$ & Y & 124 \\
\hline $7^{\star}$ & 2.646 & $\mathrm{Y}$ & $\mathrm{N}$ & 128 & 3.087 & $\mathrm{~N}$ & Y & 128 \\
\hline $8^{*}$ & 2.205 & $Y$ & $\mathrm{~N}$ & 125 & 2.646 & $\mathrm{~N}$ & $Y$ & 115 \\
\hline
\end{tabular}

${ }^{\star}$ Cadaver heads that underwent reimpaction.

$\mathrm{IOP}=$ intraocular pressure; N/A = not applicable.

Table II details the reimpaction of control side orbits in four of the cadaver heads along with their experimental side comparisons. Controls in two of the cadavers had already tolerated higher energy impactions than the experimental sides, and two of the cadavers (cadavers 1 and 2) had unfortunately been cremated before reimpaction was possible. A total of six cadavers had energy delivered to the control side sinuses that was equivalent to or surpassed the energy delivered to the contralateral experimental side. None of the six cadavers sustained globe ruptures on the control side despite the higher energies delivered, including the four cadavers that were reimpacted after the initial fractures were identified. These heads were not rescanned, but all of the floor fractures appeared to worsen by visual inspection and/or palpation. The average energy delivered to the six control sides for comparison was $2.04 \pm$ $0.57 \mathrm{~J}$. IOPs measured during these control side experiments averaged $116.8 \pm 6.1 \mathrm{~mm} \mathrm{Hg}$.

\section{DISCUSSION}

Numerous theories have been proposed for the function of the paranasal sinuses, none with empiric proof. Scientists are using fossil records to study the evolutionary development of the paranasal sinuses. Rae and Koppe $^{1}$ studied ape development extensively, and according to their analysis in some species the frontal sinus may in fact be an outgrowth of the sphenoid sinus; however, in other primates this is not the case. In either case, the true evolutionary value of the paranasal sinuses is still unclear.

Some theories have been discarded based on scientific findings. ${ }^{1-5}$ Examples are the theories of mucus secretion, voice resonance, and air reservoirs. Mucus secretion as a reason for paranasal sinus existence was discredited based on the histological evidence of decreased mucus gland populations in sinus respiratory epithelium compared with nasal respiratory epithelial linings. ${ }^{3,4}$ The suggestion that the sinuses function as a voice resonance chamber is made less likely by the anatomy of giraffes and lions. ${ }^{2-4}$ Giraffe sinuses are large, but they produce weak nonresonant vocalizations. Lions have small sinuses but produce large roars.
There are many remaining anthropologic developmental theories: 1) the reduction of cranial weight, 2) accommodation for the developmental increase in body:head ratio size, 3) the replacement of functionless bone, 4) interspaces between developing cranial architecture, 5) aids in ventilation, 6) shock absorption spaces, 7) insulation from environmental heat or cold extremes, and 8) vestigial olfactory organs. These thus far have been refuted, but not disproven. ${ }^{1-5}$ The possibility that the sinuses serve to improve nasal immunity and provide an antimicrobial benefit is intriguing. ${ }^{5}$

The hypothesis of this study is that in homo sapiens, the paranasal sinuses serve as crumple zones to protect surrounding vital structures, an explanation that from an evolutionary standpoint would at least provide the potential of a survival benefit.

The magnitude of current day trauma forces is multiple orders of magnitude greater than in days past, yet despite the high energy/high impact injuries that are sustained, there still exists a high degree of sparing of the optic nerves, globes. and viscera, with low published rates of vision loss ranging from $0.8 \%$ to $9 \% .{ }^{11-18}$ This is consistent with the crumple zone theory. For example, with severe impacts against the nasal root, a naso-ethmoid complex fracture frequently occurs; the thick glabellar bone telescopes through the thin lamina papyracea and ethmoid sinuses, thereby minimizing the transmission of energy and impact force to the optic nerve, which is also protected by the thick bone of the optic canal.

Mackinnon et al.'s ${ }^{12}$ recent Australian review of 11 years of facial fracture injuries detailed a total of 2,516 patients with facial fractures, in whom $13 \%$ had ocular complications but only $0.8 \%(\mathrm{n}=19)$ had vision impairment, and specifically $0.2 \%(\mathrm{n}=5)$ sustained globe rupture. This study also detailed that $47 \%$ of the patients studied were involved in motor vehicle accidents, and that most patients $(15 / 19,79 \%)$ with vision impairment had Glasgow coma scores (GCS) of 14 or better, suggesting a low incidence of cranial injuries. Frontal sinus fractures and collapse may also serve to protect the anterior fossae from some direct frontal trauma.

Ansari $^{11}$ reported similar numbers in a 19 -year review of Iranian patients suffering facial fractures. Of the 2503 patient studied retrospectively, $22 \%(\mathrm{n}=550)$ 
had orbital fractures, with $3.31 \%(\mathrm{n}=83)$ having ocular injuries, and 39 patients $(1.56 \%)$ having visual impairment or blindness. Two patients had ruptured globes, and 11 had eyeball destruction, or a $0.5 \%$ rate (13/2503).

Compared to the time period through which humans have evolved, the modern day environment is one of high velocity and high-impact injuries, which might make the value of the paranasal sinuses as crumple zones less apparent. High-energy traumas with the resultant increase in brain injuries make it easy to ignore the possible survival benefit of the sinus crumple zones. However, when examining the lower energy traumas, which would have been more typical of injuries that occurred during most of human evolution, the likelihood that the paranasal sinuses provided some protective benefit against visceral injuries that might cause blindness and/or brain injury becomes more salient.

The blowout fracture seems to be an appropriate paradigm for analysis of the potential benefits during our evolutionary past, because most pure blowout fractures are from lower energy impacts (such as fists and baseballs).

Further data regarding orbital injuries and pure blowout fractures has been recently published involving 10 years of retrospectively reviewed data $^{14}$ Of the pure orbital blowout fractures, the globe injury rate directly attributed to trauma was calculated as $22 \%$. The most common injuries were commotio retinae (retinal contusion) $(8.75 \%)$, traumatic mydriasis $(7.9 \%)$, and traumatic iritis $(6.25 \%)$. Only two of 225 patients $(0.83 \%)$ suffered globe ruptures. In this review of orbital injuries, $24 \%$ were pure blowout fractures and $76 \%$ mixed orbital fractures. Despite the large number of blowout fractures reviewed, very few of these patients sustained blindness as a result of trauma. This clinical finding provides modern day evidence of a protective benefit of human craniomaxillofacial anatomy.

To compare the existing facial shape with and without sinuses as crumple zones, the experimental paradigm was created by eliminating the sinuses on one side of the cadaver specimens. The method selected to do this was to fill the sinuses with a solid cement to eliminate the crumple zones without altering the shape of the craniofacial architecture. Because the anatomic shape of the midface is necessary to support the maxillary dentition allowing for the mastication of food, it was not justified to change the facial shape for this paradigm. The solid bony buttresses of the midface appear to be necessary to support effective mastication; however, the thinning of the bone between the buttresses only serves to weaken the facial bony architecture and therefore provides no obvious bony benefit.

The crumple zone concept elegantly explains this otherwise enigmatic structural development in human facial evolution. The solid supports provide adequate strength for physiologic function, whereas the weaker bone between these supports allows the midface to shear and crumple in response to impact.

A controlled experimental model with consistently repetitive delivery of trauma is needed to determine any derived benefit. Furthermore, due to species differences in globe thickness ${ }^{19}$ and the cranial and paranasal anatomy, ${ }^{1,2,4,20,21}$ a live experimental animal model, in addition to being expensive and difficult to effectively execute, would be limited for comparison and extrapolation purposes to humans. Therefore, a reproducible trauma model using fresh human cadavers was developed. For the injury mechanism, a direct drop impact was utilized due to the ease of implementation, analysis, and reproducibility. Fresh heads were used to avoid possible alterations in the biomechanical properties of the tissues that may occur as a result of the fixation process.

In addition, there also needed to be available methods to access the sinuses and obliterate them without otherwise destroying the native anatomy. The advent of endoscopic sinus techniques combined with the availability of injectable cements with properties that mimic bone provided the technological advances that made this feasible. Orbital fractures were chosen because of their reproducibility and the relative accessibility of the surrounding sinuses for the injection of cement. It is also an excellent clinical paradigm for the crumple zone hypothesis. Recent published literature regarding orbital fracture forces and mechanisms also detailed the approximate energy needed to induce orbital fractures utilizing the hydraulic mechanism. ${ }^{20-27}$

The etiology of orbital blowout fractures is controversial and has been debated for years. Two main mechanisms proposed for these fractures are the buckling theory and the hydraulic theory. The buckling theory suggests that orbital floor fractures are induced by trauma to the inferior orbital rim. According to this theory, the rim bends in response to the force causing the floor to buckle and fracture. If the force is not strong enough to fracture the rim, it returns to its initial preinjury position leaving the blowout fracture in the orbital floor. The hydraulic theory suggests that trauma to the globe is dispersed throughout the orbit with a resultant fracture of the orbital floor and in some cases the medial wall depending upon the amount of energy transmitted. Definitive proof for the hydraulic mechanism has been seen both clinically in case reports of patients subjected to barotrauma from blast injuries ${ }^{28,29}$ and experimentally in both human and animal models. ${ }^{20-27}$ Evidence for the buckling theory has also been documented in human and animal models. ${ }^{21-26}$ In this study, for the sake of simplicity and reproducibility, the paradigm of direct globe trauma was selected.

The experiments were performed with the lids closed. Other groups have described experiments both with the eyelids opened ${ }^{20,21}$ and closed. ${ }^{27}$ In trial drops on a fixed cadaver head, and one side of a fresh cadaver, drops on the globe with open eyelids resulted in globe ruptures at the lowest energies (1.18 J generated by a $200-\mathrm{g}$ rod dropped from a $60-\mathrm{cm}$ height) with ruptures that occurred directly in the impact footprint of the impacting rod. Semilunar ruptures were seen directly where the impact occurred. When the impact was directed onto closed eyelids, this did not occur. Therefore, the eyelids were sewn together with a temporary tarsorrhaphy and the tip of the impaction rod was placed inside the cut fingertip of a nitrile glove. Repeat testing starting at this 
lowest energy drop did not result in globe ruptures. Globe instrumentation consisted of a 30-gauge needle inserted temporally to monitor intraimpact IOPs and a force transducer inserted inferior to the globe.

In the definitive experiments, ruptures occurred either equatorially or posteriorly, but none on the anterior surface of the globe in the direct line of impact. In globes that underwent pressure monitoring, no leakage of fluid or vitreous occurred at the insertion site of the needle. Ruptures occurred distant from the insertion site, which was placed temporally when used. These findings are consistent with other publications regarding experimental globe rupture. ${ }^{30}$

All of the orbits with obliterated sinuses subjected to trauma sustained globe ruptures. Impaction on the control orbits did not induce ruptures; instead, only orbital floor fractures occurred. Most of the floor fractures occurred in the medial orbital floor as expected and were similar to those seen in prior experimental studies of orbital floor fractures. ${ }^{21,25,26,31,32}$

No medial wall orbital fractures were created in the experiments; only orbital floor fractures were found. This could be explained by the low energy of impact that was used to induce the fractures, $<3 \mathrm{~J}$ in most fractures. This has been seen in other studies involving human cadavers. Rhee's group ${ }^{27}$ utilized an increasing trauma force in fresh human cadavers and induced orbital floor fractures at a lower energy (at or above $2.9 \mathrm{~J}$ ) and medial wall fractures were seen experimentally with energies of $4.9 \mathrm{~J}$ or greater, and no globe ruptures were reported.

Other groups that have studied orbital fractures also demonstrated that similarly low magnitude energies were needed to create orbital floor fractures. Ahmad et al. $^{24}$ directly measured the energy to fracture an orbital floor in human cadavers at 1.22 J. Gilliland ${ }^{21}$ in an in vivo caprine model induced orbital blowout fractures with impact energies averaging $2.6 \mathrm{~J}$. Warwar, ${ }^{25,26}$ in experiments involving direct impacts on the bony orbital floors of exenterated human cadaver orbits, required energy only on the order of $70 \mathrm{~mJ}$. Gilliland et al. ${ }^{21}$ suggested that the actual impact forces seen in the clinical situation may be higher than those seen in the laboratory; however, these are only estimates and because the impacts typically resulted in blowout fractures only and not blindness, these findings do not contradict the findings in the present study.

Green's group ${ }^{20}$ induced orbital fractures in an in vivo primate model, the Macaca monkey. They induced orbital fractures at similar experimental energy levels, and they noted a threshold of $2.08 \mathrm{~J}$. But interestingly, in their study they also had a high rate $(23 \%)$ of globe ruptures at these equivalent energy levels. Other authors in addition to Green's group ${ }^{1,20,21}$ have mentioned that the Macaca monkey does not have ethmoid sinuses, only maxillary sinuses, leading to specula$\operatorname{tion}^{14,20,21}$ that this caused the high rate of ruptures. It is, of course, also possible that in this monkey species the globe rupture threshold is lower, and possibly similar to the fracture threshold of the orbital floor. Thus, evolutionary divergence may have led to this particular species not developing the beneficial crumple zone hypothesized in the current paper to have developed in humans. Both globe ruptures and orbital floor fractures in this particular monkey study occurred at similar energy levels. Green's group also noted that in one monkey, the periorbital bone was thicker, possibly due to maxillary sinus hypoplasia, and this increased bone thickness likely prevented a floor fracture, although a globe rupture did not occur either in this monkey.

The current study demonstrated that when the weak orbital floor and medial orbital walls were eliminated by the filling of the paranasal sinuses with cement, trauma delivered to the orbit resulted in globe rupture in every case. On the normal control side, no globe ruptures occurred with similar and even higher energy impacts. This is consistent with the clinically seen blowout fracture. It seems logical to conclude that this anatomic development, i.e., the thin sinus bone crumpling to protect the integrity of the globe, would impart a survival advantage over the situation with solid sinuses and thus unyielding orbital walls.

Similar peak intraocular pressures were measured in the globes on the experimental and control sides. No pattern of higher IOPs was seen in the orbital floor fracture side compared to the globe rupture side (116.8 \pm $6.1 \mathrm{~mm}$ vs. $121.5 \pm 6.0 \mathrm{~mm} \mathrm{Hg}$, respectively). When a critical energy threshold was reached, one of two things happened: either the floor fractured or the globe ruptured. In the normal control side, the pressure relief valve of the orbital floor was available for fracture, i.e., the crumple zone was protective. Experimentally obliterated sinuses did not allow for this pressure outlet through the thin orbital floor (or medial walls of the orbit due to the ethmoid sinus obliteration), and thus the globes ruptured.

There is little data available on the force needed for globe rupture induced by blunt trauma. Nonetheless, it is common knowledge that in clinical practice globe ruptures remain uncommon despite the relative frequency of occurrence of nasoethmoid complex, orbital, maxillary, and zygomaticomaxillary complex fractures.

The average age of the cadavers used in this experiment $(76.1 \pm 12.2$ years $)$ is higher than that of typical orbital blowout trauma patients. However, past studies on fracture patterns ${ }^{21}$ have not indicated any effect of age on bone fracture biomechanics. Recent studies ${ }^{33}$ on human globes found evidence of age-induced weakening of the globe. However, this study utilized intraspecimen controls, which should minimize that concern. In addition, the IOPs at globe rupture were similar for all specimens in this study regardless of age.

One of the technical problems encountered in the study was the incomplete filling of the sinuses with cement. These unfilled areas were evaluated on the CT scans and were found to involve regions distant from the classic blowout fracture sites and were therefore unlikely to have an impact on the study results. In fact, the areas of the orbital floor that fractured on the control (normal) sides were fully supported by cement in each of the experimental sides. Thus, the critical area of the orbital floor was reinforced so that the crumple zone was effectively eliminated. 
Regarding the number of specimens studied, 100\% globe rupture occurred on the experimental side, and $100 \%$ of the orbital floor fractures were induced on the contralateral control sides. Therefore, the additional cost of increasing numbers of cadavers seemed unwarranted.

There was one instance (cadaver 4) in which the energy that caused globe rupture on the experimental side was lower than the energy required on the control side to fracture the floor, yet the control side globe did not rupture. There is no particular explanation for this occurrence. However, it is possible that invisible microfractures in the unsupported orbital bone may have allowed for this higher impact tolerance.

The presence of a very thin orbital floor that easily fractures seems to provide a protective benefit for the globe, so that globe rupture is less likely to occur when the globe is traumatized. It seems reasonable to conclude that in the clinical setting, the infrequency of globe injury in the presence of blowout fractures is likely the result of this now experimentally demonstrated protective effect provided by the paranasal sinuses and the thin intervening bone between the sinuses and the orbit. Although it is impossible to conclude that this structural benefit assures evolutionary development, particularly in the absence of anthropological support, ${ }^{1,3,5}$ it is certainly suggestive. Furthermore, Rae and Koppe (citing Gould and Vrba) ${ }^{1}$ point out that an existing benefit of a structure does not assure that it was responsible for its evolution. Nonetheless, the findings remain intriguing.

This crumple zone theory thus serves to explain not only the potential survival benefit provided by the paranasal sinuses in humans, but it also explains the unusual facial architecture of solid pillars of bone that provide structural support for mastication that are surrounded by otherwise unexplainably thin bone that provides little structural integrity to the facial skeleton. This concept can be extended to other areas of the facial skeleton, with the thin lamina papyracea and ethmoid sinuses providing potential protection for the globes, optic nerves, olfactory elements and anterior fossae, the frontal sinuses protecting the frontal lobes, the sphenoid sinuses potentially protecting the optic nerves, carotid arteries, cavernous sinuses and surrounding brain structures, and the maxillary sinuses potentially protecting the middle cranial fossae.

\section{CONCLUSION}

This study demonstrates that when trauma is directed to the globe, the thin orbital floor fractures preferentially into the maxillary sinus, thereby protecting the globe from rupture. When the ethmoid and maxillary sinuses were eliminated, similar trauma caused globe ruptures to occur. These findings support the role of the sinuses as a protective crumple zone for the orbit.

\section{Acknowledgments}

The authors wish to thank Dr. Julie Hasenwinkel, Assistant Professor and Program Director of Bioengineering, Syracuse University, Syracuse, New York for providing bone cement and assistance; and Ms. Marci Guyer for her invaluable assistance with preparation of this manuscript.

\section{BIBLIOGRAPHY}

1. Rae TC, Koppe T. Holes in the head: Evolutionary interpretations of the paranasal sinuses in catarrhines. Evol Anthropol 2004;13:211-223.

2. Takahashi $R$. The formation of the human paranasal sinuses. Acta Otolaryngol 1983;408(suppl):1-28.

3. Blaney SPA. Why paranasal sinuses? J Laryngol Otol 1990; 104:690-693.

4. Rhys-Evans PH. The paranasal sinuses and other enigmas: an aquatic evolutionary theory. $J$ Laryngol Otol 1992; 106:214-225.

5. Keir J. Why do we have paranasal sinuses? J Laryngol Otol 2009;123:4-8.

6. MSN Encarta. Crumple zone. [Encarta Web site]. Available at: http://encarta.msn.com/encyclopedia_762505333/crumple_ zone.html. Accessed April 15, 2009.

7. Manson PN, Hoopes JE, Su CT. Structural pillars of the facial skeleton: an approach to the management of Le Fort fractures. Plast Reconstr Surg 1980;66:54-62.

8. Stanley RB Jr. Reconstruction of the midfacial vertical dimension following LeFort fractures. Arch Otolaryngol 1984;110:571-575.

9. LeFort R. Experimental study of fractures of the upper jaw [translated from French]. Plast Reconst Surg 1972;50: 600-607.

10. Kellman RM. Maxillofacial trauma. In: Cummings CW, Flint PW, Harker LA, et al., eds. Cummings Otolaryngology Head \& Neck Surgery. 4th ed. Philadelphia, PA: Mosby, Inc.; 2005:602-638.

11. Ansari MH. Blindness after facial fractures: a 19-year retrospective study. J Oral Maxillofac Surg 2005;63:229-237.

12. MacKinnon CA, David DJ, Cooter RD. Blindness and severe visual impairment in facial fractures: an 11 year review. Br J Plast Surg 2002;55:1-7.

13. Dancey A, Perry M, Silva DC. Blindness after blunt facial trauma: are there any clinical clues to early recognition? $J$ Trauma 2005;58:328-335.

14. He D, Blomquist PH, Ellis E. Association between ocular injuries and internal orbital fractures. J Oral Maxillofac Surg 2007;65:713-720.

15. Linnau KF, Hallam DK, Lomoschitz FM, Mann FA. Orbital apex injury: trauma at the junction between the face and the cranium. Eur J Radiol 2003;48:5-16.

16. Pappachan B, Alexander M. Correlating facial fractures and cranial injuries. J Oral Maxillofac Surg 2006;64: 1023-1029.

17. Cruz AAV, Eichenberger GCD. Epidemiology and management of orbital fractures. Curr Opin Ophthalmol 2004;15: 416-421.

18. Plaisier BR, Punjabi AP, Super DM, Haug RH. The relationship between facial fractures and death from neurologic injury. J Oral Maxillofac Surg 2000;58:708712 .

19. Stitzel JD, Hansen GA, Herring IP, Duma SM. Blunt trauma of the aging eye: injury mechanisms and increasing lens stiffness. Arch Ophthalmol 2005;123:789-794.

20. Green RP Jr, Peters DR, Shore JW, Fanton JW, Davis H. Force necessary to fracture the orbital floor. Ophthal Plast Reconstr Surg 1990;6:211-217.

21. Gilliland GD, Gilliland G, Fincher T, Harrington J, Gilliland JM. Assessment of biomechanics of orbital fracture: a study in goats and implications for occuloplastic surgery in humans. Am $J$ Ophthalmol 2005;140: 868-876.

22. Waterhouse N, Lyne J, Urdang M, Garey L. An investigation into the mechanism of orbital blowout fractures. $\mathrm{Br}$ J Plast Surg 1999;52:607-612.

23. Smith B, Regan WF Jr. Blow-out fracture of the orbit: mechanism and correction of internal orbital fracture. Am J Ophthalmol 1957;44:733-739.

24. Ahmad F, Kirkpatrick NA, Lyne J, Urdang M, Waterhouse N. Buckling and hydraulic mechanisms in orbital blowout 
fractures: fact or fiction? J Craniofac Surg 2006;17: 438-441.

25. Bullock JD, Warwar RE, Ballal DR, Ballal RD. Mechanisms of orbital floor fractures: a clinical, experimental, and theoretical study. Trans Am Ophthalmol Soc 1999;97: 87-110; discussion 110-113.

26. Warwar RE, Bullock JD, Ballal DR, Ballal RD. Mechanisms of orbital floor fractures: a clinical, experimental, and theoretical study. Ophthal Plast Reconstr Surg 2000;16: 188-200.

27. Rhee JS, Kilde J, Yoganadan N, Pintar F. Orbital blowout fractures: experimental evidence for the pure hydraulic theory. Arch Facial Plast Surg 2002;4:98-101.

28. Suzuki H, Furukawa M, Takahashi E, Matsuura K. Barotraumatic blowout fracture of the orbit. Auris Nasus Lar$y n x$ 2001;28:257-259.
29. Shamir D, Ardekian L, Peled M. Blowout fracture of the orbit as a result of blast injury: case report of a unique entity. J Oral Maxillofac Surg 2008;66:14961498.

30. Haug RH, Haghighi DB, Barber JE. An experimental evaluation of traumatic globe rupture. J Oral Maxillofac Surg 1998;56:1275-1280.

31. Jackson IT. Classification and treatment of orbitozygomatic and orbitoethmoid fractures. The place of bone grafting and plate fixation. Clin Plast Surg 1989;16:77-91.

32. Fujino T, Sugimoto C, Tajima S, Moribe Y, Sato TB. Mechanism of orbital blowout fracture: II. Analysis by high speed camera in two dimensional eye model. Keio J Med 1974;23:115-124.

33. Vinger PF. Understanding eye trauma through computer modeling. Arch Ophthalmol 2005;123:833-834. 